\title{
Directly Synthesized Strong, Highly Conducting, Transparent Single-Walled Carbon Nanotube Films
}

\author{
Wenjun Ma, ${ }^{\dagger, \perp}$ Li Song,,$^{\S}$ Rong Yang, ${ }^{\ddagger, \perp}$ Taihua Zhang, ${ }^{\ddagger}$ Yuanchun Zhao, $, 1, \perp$ \\ Lianfeng Sun," Yan Ren, ${ }^{\dagger, \perp}$ Dongfang Liu, ${ }^{\dagger, \perp}$ Lifeng Liu, ${ }^{\dagger, \perp}$ Jun Shen, ${ }^{\dagger, \perp}$ \\ Zhengxing Zhang, ${ }^{\dagger, \perp}$ Yanjuan Xiang, ${ }^{\dagger, \perp}$ Weiya Zhou, ${ }^{\dagger}$ and SiShen $\mathrm{Xie}^{\star, \dagger}$
}

Beijing National Laboratory of Condensed Matter, Institute of Physics, Chinese Academy of Sciences, Beijing 100080, P.R. China, State Key Laboratory of Nonlinear

Mechanics, Institute of Mechanics, Chinese Academy of Sciences, Beijing 100080,

P.R. China, Center for Nanoscience and Department für Physik, Ludwig-Maximilians-Universität München, Geschwister-School-Platz 1, 80539 München, Germany, National Center for Nanoscience and Nanotechnology, Chinese Academy of Sciences, Beijing 100080, P.R. China, and Graduate School of the Chinese Academy of Sciences, Beijing 100080, P.R. China

Received April 18, 2007; Revised Manuscript Received June 15, 2007

\begin{abstract}
We report the direct synthesis of strong, highly conducting, and transparent single-walled carbon nanotube (SWNT) films. Systematically, tests reveal that the directly synthesized films have superior electrical and mechanical properties compared with the films made from a solution-based filtration process: the electrical conductivity is over $2000 \mathrm{~S} / \mathrm{cm}$ and the strength can reach $360 \mathrm{MPa}$. These values are both enhanced by more than 1 order. We attribute these intriguing properties to the good and long interbundle connections. Moreover, by the help of an extrapolated Weibull theory, we verify the feasibility of reducing the interbundle slip by utilizing the long-range intertube friction and estimate the ultimate strength of macroscale SWNTs without binding agent.
\end{abstract}

Because of their optical transparency and unique electric properties together with mechanical flexibility, film-like single-walled carbon nanotubes (SWNTs) are attractive not only for fundamental researches but also potential applications. For example, large optical nonlinearity, ${ }^{1}$ subpicosecond optical response $\mathrm{e}^{2,3}$ and bolometric infrared photoresponse $\mathrm{e}^{4}$ have been observed in SWNT films, and the feasibilities of using SWNT films or networks as sensors, ${ }^{5,6}$ diodes, ${ }^{7}$ and field effect transistors ${ }^{8}$ have already been demonstrated. Recently, highly conducting transparent SWNT films (tSWNTs) have also been fabricated by a kind of controlled filtration-deposition process, ${ }^{9}$ which could be used as transparent electrodes for GaN/InGaN or flexible organic lightemitting diodes. ${ }^{10,11}$ However, almost all of the above reports focused on the post-treated SWNT films, which are obtained through solution-based filtration processes. As known, to

* Corresponding author. E-mail: ssxie@aphy.iphy.ac.cn. Telephone: +86-10-82649081. Fax: +86-10-82640215.

'Beijing National Laboratory of Condensed Matter, Institute of Physics.

State Key Laboratory of Nonlinear Mechanics, Institute of Mechanics.

$\S$ Center for Nanoscience and Department für Physik, Ludwig-Maximilians-Universität München.

"National Center for Nanoscience and Nanotechnology.

${ }^{\perp}$ Graduate School of the Chinese Academy of Sciences. obtain high conductivity, SWNTs must be well purified and dispersed from sootlike morphology, which usually costs several days and leaves unrecyclable chemical residues. Besides, the comparative low strength of these films is one of the challenges for their applications, especially in the field of high-strength enforcement sheets.

Here we report the direct synthesis of strong, highly conducting, and transparent films through a further developed floating catalyst CVD (FCCVD) technique that is based on the methods of producing large-scale nonwoven SWNTs. ${ }^{12}$ As catalyst source, ferrocene/sulfur powder is heated to 65$85^{\circ} \mathrm{C}$ and flowed into a reaction zone by the mixture of $1000 \mathrm{sccm}$ argon and $1-8 \mathrm{sccm}$ methane. The growth rates of the films are mainly determined by the sublimation rate of the catalysts. Under typical conditions, after $30 \mathrm{~min}$ growth, thin films with a thickness of $100 \mathrm{~nm}$ will form in the high-temperature zone (over $600{ }^{\circ} \mathrm{C}$ ) of the quartz tube and can be easily peeled off. This type of large-area freestanding film can be easily handled for further researches. Raman scattering and HRTEM images show that most CNTs in the films are single-walled carbon nanotubes. In this paper, we systematically investigated the properties of the directly 

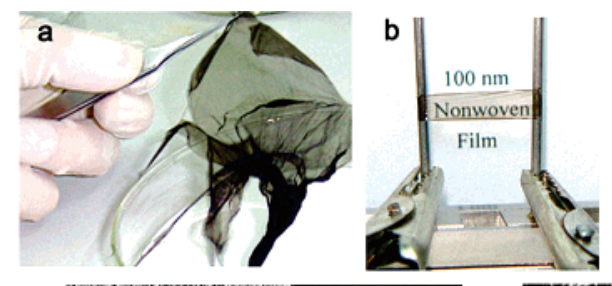

C Homogenous Film transparent Inhomogenous Film transparent

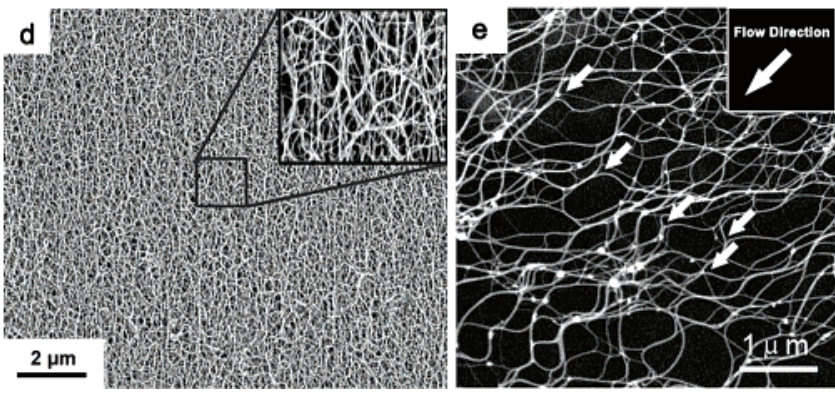

Figure 1. (a) Photo image of an as-grown $250 \mathrm{~nm}$ thick film. (b) A transparent $100 \mathrm{~nm}$ thick film freely stands between metallic pillars. (c) A $150 \mathrm{~nm}$ thick homogeneous (upper one) and inhomogeneous films. The importance of homogeneity is obvious. (d) Large-scale SEM image of a $250 \mathrm{~nm}$ thick film. The inset image is taken at higher magnification. (e) SEM image of SWNT network in a single layer. The white arrows in the image point out the Y-type junctions and the flow direction.

synthesized films with serials of optical, electrical, and mechanical tests.

To obtain high-quality transparent films, several crucial improvements have been made compared with an earlier preparation procedure: (i) the growth rates are well controlled by precisely controlling the sublimation rates of the catalysts, (ii) the impurities in the as-grown films are further reduced compared with our earlier results, (iii) the homogeneity of the films is ensured by the intentionally stabilized gas flow in the quartz tube. As seen in Figure 1a,b, the homogeneity of the film is good enough in a typical $5 \times 10$ $\mathrm{cm}^{2}$ area to reach optical transparency. If the films are not well homogeneous, due to the severe diffusion, they will lose high transparency even if they are thin enough (Figure 1c). Figure 1d is the SEM image of a $200 \mathrm{~nm}$ thick as-grown film, and we can see SWNTs are homogeneously distributed and entangled with each other, and impurities are at a low level. Because the as-grown films are not compact, the thicknesses are of meaning only for the densified films. We sliced a piece from each of the films and densified them by ethanol on silicon substrates and then measured the thicknesses by atomic force microscopy (AFM) at step edges. Serial validation experiments have shown that the thicknesses determined by this method are reliable, so all the thickness values referred to in this paper are obtained by this method although most other types of tests are based on the as-grown samples. The density of the densified film is $1.3 \pm 0.1 \mathrm{~g} / \mathrm{cm}^{3}$, from which we can infer the free volume in the densified films, is about 20-30\% (see Supporting Information).

Figure 2a shows the transmittance spectrum for the asgrown freestanding films with different thickness, and the spectral range covers from ultraviolet to the mid-infrared region. For $100 \mathrm{~nm}$ thick film, which is the thinnest film we can intactly peel from the wall of the quartz, the transmittance in the visible region of the spectrum is over $65 \%$. When the films are coated on the other substrate and densified, the transmittance will increase about $5-10 \%$ due to a denser structure and smoother surface. The absorption peaks in the rage of $600 \mathrm{~nm}$ to $3 \mu \mathrm{m}$ come from the electronic transitions of the semiconductive and metallic SWNTs, respectively, and according to that, the diameters of the nanotubes in the films could be assigned to between 1.2 and $2.0 \mathrm{~nm} .{ }^{13}$ This diameter distribution is consistent with Raman spectrum (see Supporting Information).

The measurements of the sheet electrical resistances of the as-grown films are carried out on a two-pillar metallic holder (see Figure 1b) and recorded by a Keithley 4200 system. Although some contact resistance is introduced in this kind of two-lead measurements, the measured sheet resistances are intriguingly low (Figure $3 b$ ). The sheet resistance is $50 \Omega$ /square for the $100 \mathrm{~nm}$ thick film, which has transmittance greater than $70 \%$ on substrate. Considering that the tested films have not undergone any chemical treatment, which usually increases the conductivity several times, ${ }^{14}$ this value is remarkable low for such transparent film. To decrease the influence of the thickness $(t)$ uncertainty, we obtain the electrical conductivity $(\sigma)$ by fitting the sheet resistance vs thickness curve using the formula $\sigma$ $=1 / R t$. The best fitted conductivity is $2026 \mathrm{~S} / \mathrm{cm}$, over 60 times larger than that of unpurified HiPCO SWNTs transparent films and near 3 times of the purified arc-discharge SWNTs films. ${ }^{11}$ This value is amazingly low because, compared with as-produced HiPCO SWNTs, their purities are similar (75-85 wt \%).

Although the axial conductivity of a SWNT rope can reach $10000-30000 \mathrm{~S} / \mathrm{cm},{ }^{15}$ conductivity in films or networks are usually 1 or 2 orders lower. It is believed that the conductivity of the films is dominated by the intertube junctions. Purifying or compacting the films have been proved favorable to increase the conductivity to a certain degree. ${ }^{9}{ }^{14}$ In our case, we found the unique structure results in the high conductivity: (i) The bundles in the films are not randomly distributed but preferentially aligned by the flow when they are growing in the reaction zone. Figure 1e is the SEM image of a single layer of bundles peeled off from a $100 \mathrm{~nm}$ thick film. It indicates that almost all the Y-type junctions are along the flow direction. The preferential orientation results in anisotropically mechanical property: the strength in the flow direction is much higher than that in the vertical direction. Because of the anisotropic strength, all the films suspended between two metallic pillars are mounted along the preferential direction, which effectively enhances the conductivity as revealed before. ${ }^{16}$ (ii) The bundles in the films firmly connect with each other when they are growing and forming continuous preferential 2-D web at high temperature, whereas in the films made by filtration, the contacts are weak and sometimes blocked by amorphous carbon. In fact, the bondings at the junction parts are so strong that when we try to disperse the tubes in acetone, the films still keep their intactness even after hours of ultrasonication. Up to now, although many techniques have been developed to treat SWNTs, few methods are 

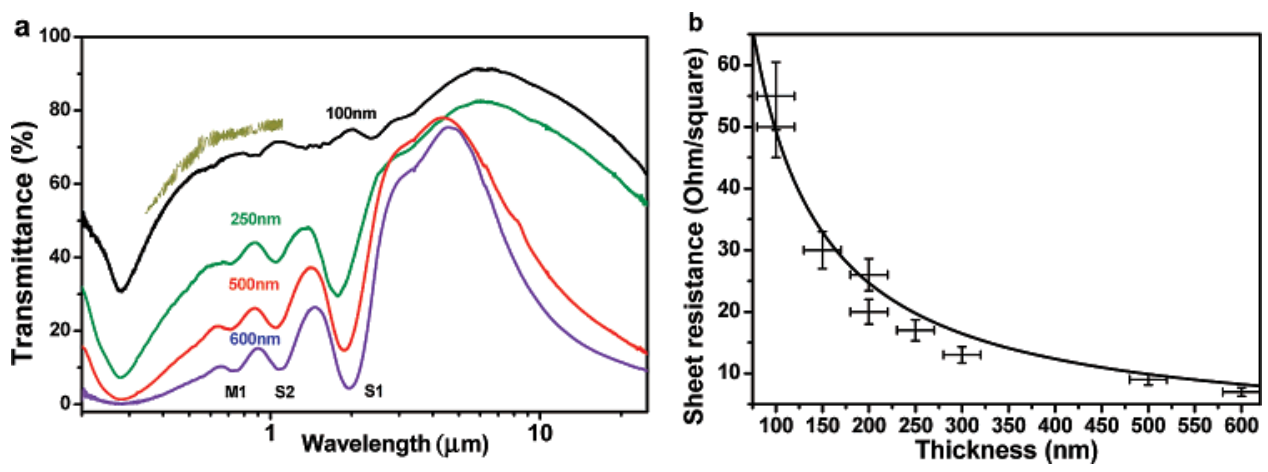

Figure 2. (a) Transmittance spectra for as-grown films with different thicknesses. The numbers above the curves notify the thicknesses, and the uppermost short curve corresponds to the $100 \mathrm{~nm}$ thick film on glass substrate. S1,S2 represent the electronic transitions for the semiconducting SWNTs in the films, and M1 represents that of the metallic nanotubes. (b) Sheet resistance vs thickness of SWNT films. The solid line is the best fitted curve according to the definition of electrical conductivity.

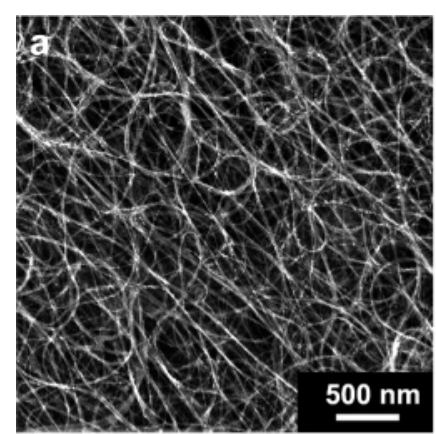

e

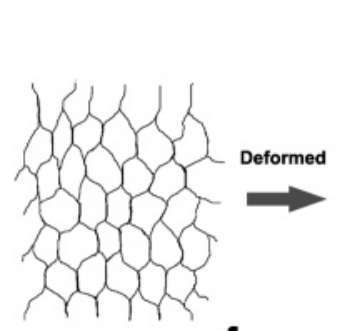

f
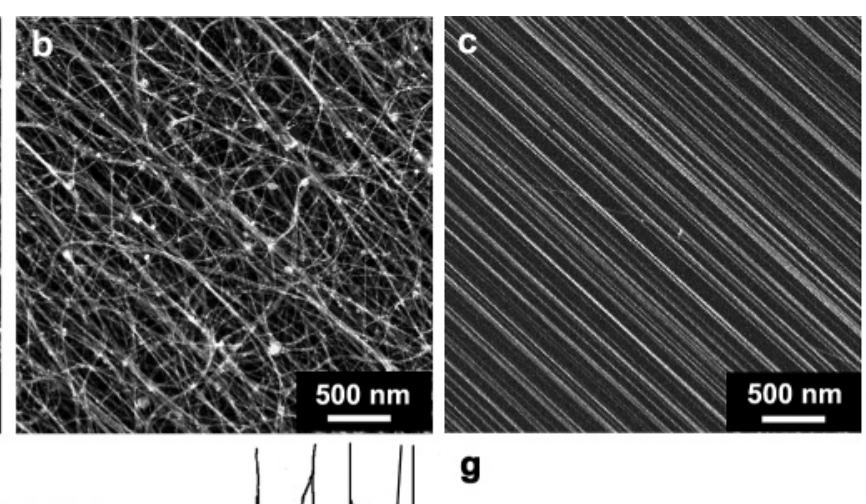

g
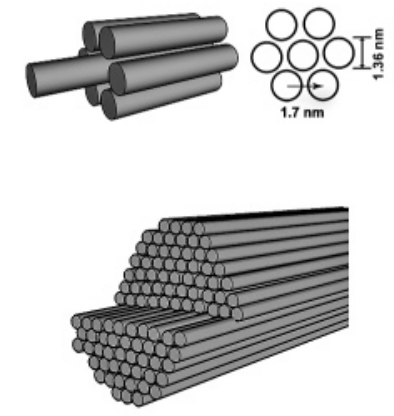
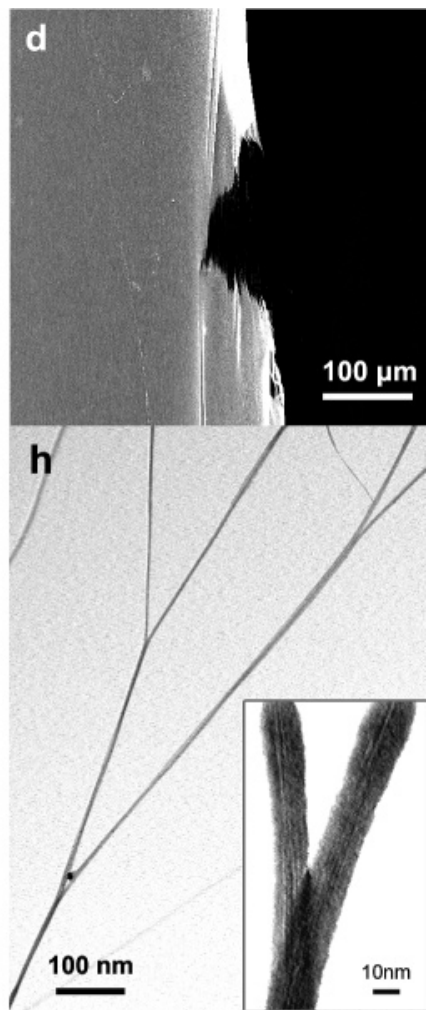

Figure 3. (a) SEM image of an unstrained film. (b) Typical SEM image of the film after $8-10 \%$ strain before broken. (c) SEM image at the breaking point. (d) Macroscopic SEM image near the breaking point. The film is going to be split. (e) Schematic diagram of the structure deformation process during extension. (f) Schematic diagram of one simplified mesh and its morphology after 33\% extension. (g) Drawing a $(10,10)$ SWNT from a $(10,10)$ bundle (upper two images), and the interbundle slipping within a $17 \mathrm{~nm}$ wide $(10,10)$ shared bundle. It is easy to count out that the area of the slipping surface is about $10 \%$ of the total outer surface for the SWNTs in a semibundle. (h) Low-magnification TEM image for some typical stretched SWNT meshes.

reported to improve the electrical contacts between bundles. Our result shows that connecting bundles into a continuous net when they are growing is an effective way to obtain better contacts and then lower sheet resistance.

Because of the preferentially oriented and firmly connected network structure, an unusual mechanical property is expected. By the use of a homemade microextensometer set inside the SEM chamber, we observed the in situ morphologies when the films were extended along the preferential direction. The changes of morphology are shown from parts a to $d$ of Figure 3, and a schematic illustration to show the deformation process for a single net layer is plotted in Figure 3e. On the basis of the observation, we suggest that when the strain is far below the strain-to-failure, the "meshes" in the networks extend continuously and homogeneously; with increasing strain, stress concentrations occur at weak places and become more and more severe; near to the stain-tofailure, extension mainly occurs at the breaking point, where meshes are destroyed and left tubes completely align. Once the breaking point develops, the concentrated stress will split the films rapidly. To clarify the load transfer mechanism, we simplify a mesh as a hexangular basic unit. As seen in Figure $3 \mathrm{f}$, the maximum extension ratio of the basic unit is $33 \%$, far higher than the typical strain-to-failure of the films 
a

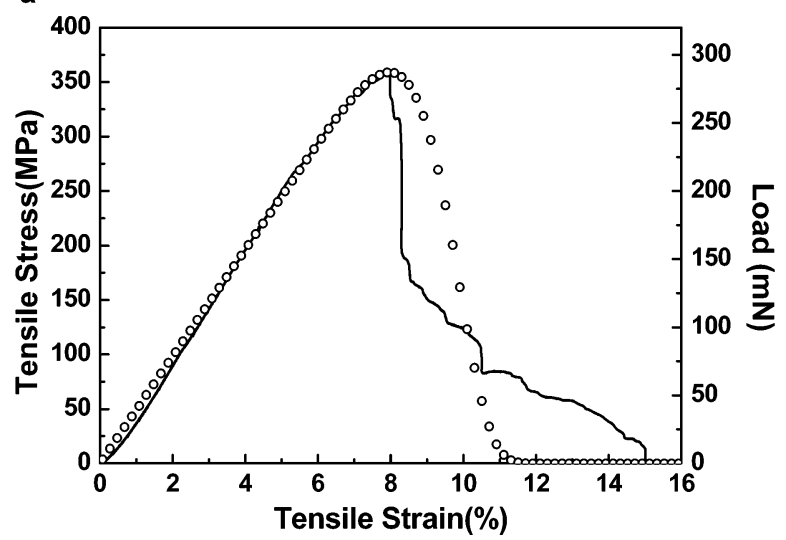

b

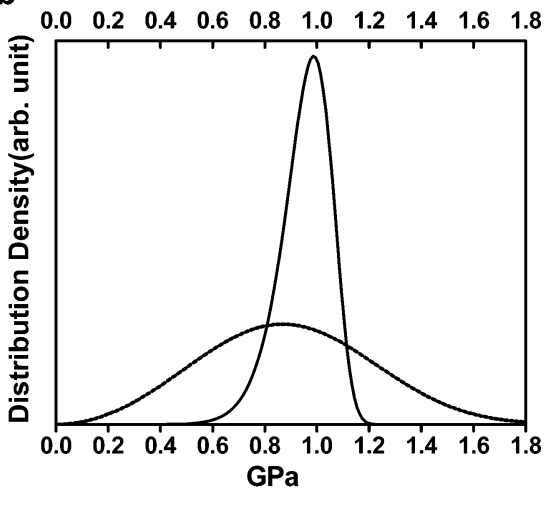

Figure 4. (a) Tested strain-stress curve for a $200 \mathrm{~nm}$ thick film (solid line), and best-fitted curve (circle points) with formula (eq 2). (b) Calculated strength distribution for the meshes in the film (solid line), and an assumed distribution with shape parameter of 3 (dot line).

$(10 \%)$. Hence we have good reason to infer the mechanical property of the macroscopic films is dominated by the basic units, meshes, rather than straight bundles, and the load is homogeneously transferred to whole film through shared bundles of adjacent meshes.

To evaluate the films' mechanical property exactly, tensile tests were performed. The as-grown films were first stuck onto center-hollow paper cards and then mounted in the tensile test apparatus (Instron 5848 microtester, load cell $5 \mathrm{~N}$ ) with cards. The sheet gauge lengths were fixed to $1 \mathrm{~cm}$, the widths vary from 3 to $5 \mathrm{~mm}$, and the extension rate was $1 \% / \mathrm{min}$. Figure 4 shows the tensile test result of a $200 \mathrm{~nm}$ thick film, and as expected, the film exhibits high tensile strength and good toughness. The tensile strength is $360 \mathrm{MPa}$, which is 30 and 10 times higher than typical bulky paper and sheets from oleum, ${ }^{17}$ respectively; the density-normalized stress is $280 \mathrm{MPa} /\left(\mathrm{g} / \mathrm{cm}^{3}\right)$, which is higher than that of undensified MWNT sheets drawn from nanotube array but lower than that of the densified ones. ${ }^{18}$ The Young's modulus is about $5 \mathrm{GPa}$. Compared with the theoretical strength of individual SWNT (37 GPa), the film strength is 2 orders lower. That is because, similar to other engineering materials, the strength of macroscale SWNTs is dominated by the stress transfer mechanism rather than individual CNT's strength.

As known, despite the high stiffness and strength of individual SWNTs, the slips between nanotube surfaces shadow the prospect of using SWNT bundles as enforcing material in composites. ${ }^{19,20}$ To resolve the "slipping problem", several routes have been proposed such as reducing the bundles' diameters, ${ }^{20}$ bridging adjacent tubes by electronbeam irradiation, ${ }^{21}$ or prolonging the contact length between tubes, ${ }^{22}$ but none of them have been proved feasible at macroscale. Now, the directly synthesized strong films with simple and uniform structure offer an opportunity to verify the last proposal.

Ruoff et al. pointed out ${ }^{22}$ that the change of the intertube's binding energy as intertube sliding occurs introduces "intertube friction". Although this type of friction force is 4 orders weaker than that of a covalent carbon bond, the accumulated force is also significant if the connected parts between bundles are long enough. According to their calculation, the friction force when drawing an individual
$(10,10)$ tube from a bundle composed of $(10,10)$ tubes is $0.58+0.71 L \times 10^{-3}(\mathrm{eV} / \AA)$, where $L$ is the contact length. For a $17 \mathrm{~nm}$ wide by $1 \mu \mathrm{m}$ long $(10,10)$ shared bundle (Figure $3 \mathrm{~g}$ ), where slipping only occurring at the interface of the semibundle, the friction force is $61 \mathrm{nN}$, the corresponding maximum stress on each semibundle is $540 \mathrm{MPa}$. In our films, the typical contact length is $1 \mu \mathrm{m}$ (Figure $3 \mathrm{~h}$ ) and average bundle diameter is $20 \mathrm{~nm}$, so it seems reasonable that the strength of the macroscopic films lies in the same scale.

However, strictly speaking, the measured strength of the macroscopic films cannot be directly used to test Ruoff's theory because the distributed meshes' strengths and local stress concentration will dramatically decrease the strength when the scale increases. To create the link between the strength of macroscopic films and that of micromeshes, we employ Weibull distribution.

Weibull distribution function is introduced by Waloddi Weibull and widely used in the last century to model material strength, times-to-failure of electronic and mechanical components or systems. When it is used to describe the mechanical property of a carbon fiber bundle, the broken possibility for each fiber in the bundle under strain $\epsilon$ could be written as:

$$
P(\epsilon)=1-e^{-L\left(\epsilon / \epsilon_{0}\right) m}
$$

where $m$ is the shape parameter thath controls the dispersion of the distribution, $\epsilon_{0}$ the characteristic strain, and $L$ the dimensionless length. The stain-stress curve of a fiber bundle can be expressed as:

$$
\sigma(\epsilon)=E \epsilon e^{-L\left(\epsilon / \epsilon_{0}\right) m}
$$

Considering the wide applicability of Weibull distribution, we tentatively employ eq 2 to describe the mechanical property of the films. For the $1 \mathrm{~cm}$ long gauge length and 2 $\mu \mathrm{m}$ long typical length of hexangular mesh, the parameter $L$ can be determined as 5000 in advance. Other parameters $m$, $\epsilon_{0}$, and $E$ could be fixed as $11.7,20.3 \%$, and $4900 \mathrm{MPa}$, respectively, from the best-fitted curve in Figure 4a. On the 
basis of the scalability of Weibull distribution, it is easy to figure out that the average strength of the meshes is 950 $\mathrm{MPa}$. Compared with the predicted mesh strength $540 \mathrm{MPa}$, this value is somewhat higher. This discrepancy may come from interlayer entanglements among different layers of networks or imply that the interbundle bondings are even stronger than the prediction. Further lengthening of shared bundles will enhance the strength to a certain degree, but admittedly there lies a limit for the enhancement. Even if the contact length is so long that the friction force is comparable to covalent bonds and the interbundle slidings are effectively prevented, the limited interbundle surface limits the transferred load through bundles. In fact, if we further consider the weakening when the dimension scales up, a reasonable upper limit for macroscale SWNTs without coagulant will not exceed $2 \mathrm{GPa}$, which is consistent with the observed values in macroscopic fibers, ropes or films..$^{23,24}$

It is also worth noting that the shape parameter is very large compared with typical value of carbon fibers (2-5). Larger shape parameter means narrower strength distribution and better flexibility, which comes from the superior flexibility of carbon nanotubes compared with carbon fibers. Figure $4 \mathrm{~b}$ shows the calculated strength distribution of the meshes, and as a comparison, distribution with a shape parameter of 3 is also shown. It is clear that most meshes break when the local stress lies between the 0.8 and $1.1 \mathrm{GPa}$. Superior flexibility and high strength together result in good toughness. Baughman et al. have shown ${ }^{25}$ single yarns spun from a MWNT array have toughness about $14 \mathrm{~J} / \mathrm{g}$, and solution-spun SWNT/PVA composite fibers $600 \mathrm{~J} / \mathrm{g}$. For our film, the toughness is $11.5 \mathrm{~J} / \mathrm{g}$, which is close to the MWNT yarns' value. Combined with the similar strength values between our SWNT films and Baughman's MWNT sheets and yarns, maybe it can be inferred that the load transfer mechanisms for macroscale SWNT and MWNT are similar to some extent.

As shown above, through a simple and environmentally friendly procedure, large-area transparent SWNT films are synthesized. For the directly grown films, firm bondings between bundles not only result in intriguing conductivity but also determine their strength. Because of the $1 \mu \mathrm{m}$ long interbundle connections in the film, the conductivity and strength both enhance over 1 order compared with the films made from solution-based filtration processes. Our results explicate that if we pay attention to the connections between SWNT bundles, we will find that there is plenty of room to exploit.

Acknowledgment. We gratefully acknowledge financial support from National Natural Science Foundation of China (grant nos. 10334060, 50572119, and 10572142), "973" National Key Basic Research Program of China (grant no. 2005CB623602), and The Key Item of Knowledge Innovation Project of Chinese Academy of Sciences (grant no. KJCX2-YW-M01). We also thank Prof. Baohua Feng,
Chaoying Wang, Prof. Gang Wang, Dr. Guangtong Liu, and Dr. Hanfu Wang for their assistance, as well as Prof. Rinzler for his enlightening suggestions.

Supporting Information Available: Details about the measurements of the films' thicknesses, estimation of the void ratio, and the Raman analysis. This material is available free of charge via the Internet at http://pus.acs.org.

\section{References}

(1) Maeda, A.; Matsumoto, S.; Kishida, H.; Takenobu, T.; Iwasa, Y.; Shiraishi, M.; Ata, M.; Okamoto, H. Phys. Rev. Lett. 2005, 94, 4.

(2) Tatsuura, S.; Furuki, M.; Sato, Y.; Iwasa, I.; Tian, M. Q.; Mitsu, H. Adv. Mater. 2003, 15, 534.

(3) Long, Y. B.; Song, L.; Li, C. Y.; Zhang, C. Y.; Wang, G. P.; Xie, S S.; Wang, L.; Fu, P. M.; Zhang, Z. G. Chem. Phys. Lett. 2005, 405, 300.

(4) Itkis, M. E.; Borondics, F.; Yu, A. P.; Haddon, R. C. Science 2006, $312,413$.

(5) Li, Z. L.; Dharap, P.; Nagarajaiah, S.; Barrera, E. V.; Kim, J. D. Adv. Mater. 2004, 16, 640.

(6) Snow, E. S.; Perkins, F. K.; Houser, E. J.; Badescu, S. C.; Reinecke, T. L. Science 2005, 307, 1942.

(7) Zhou, Y. X.; Gaur, A.; Hur, S. H.; Kocabas, C.; Meitl, M. A.; Shim, M.; Rogers, J. A. Nano Lett. 2004, 4, 2031.

(8) Snow, E. S.; Campbell, P. M.; Ancona, M. G.; Novak, J. P. Appl. Phys. Lett. 2005, 86, 3.

(9) Wu, Z. C.; Chen, Z. H.; Du, X.; Logan, J. M.; Sippel, J.; Nikolou, M.; Kamaras, K.; Reynolds, J. R.; Tanner, D. B.; Hebard, A. F.; Rinzler, A. G. Science 2004, 305, 1273.

(10) Lee, K.; Wu, Z.; Chen, Z.; Ren, F.; Pearton, S. J.; Rinzler, A. G. Nano Lett. 2004, 4, 4.

(11) Zhang, D. H.; Ryu, K.; Liu, X. L.; Polikarpov, E.; Ly, J.; Tompson, M. E.; Zhou, C. W. Nano Lett. 2006, 6, 1880.

(12) Song, L.; Ci, L.; Lu, L.; Zhou, Z. P.; Yan, X. Q.; Liu, D. F.; Yuan, H. J.; Gao, Y.; Wang, J. X.; Liu, L. F.; Zhao, X. W.; Zhang, Z. X.; Dou, X. Y.; Zhou, W. Y.; Wang, G.; Wang, C. Y.; Xie, S. S. Adv. Mater. 2004, 16, 1529.

(13) Kataura, H.; Kumazawa, Y.; Maniwa, Y.; Umezu, I.; Suzuki, S.; Ohtsuka, Y.; Achiba, Y. Synth. Mater. 1999, 103, 2555.

(14) Rinzler, A. G.; Liu, J.; Dai, H.; Nikolaev, P.; Huffman, C. B.; Rodriguez-Macias, F. J.; Boul, P. J.; Lu, A. H.; Heymann, D.; Colbert, D. T.; Lee, R. S.; Fischer, J. E.; Rao, A. M.; Eklund, P. C.; Smalley, R. E. Appl. Phys. A 1998, 67, 29.

(15) Fischer, J. E.; Dai, H.; Thess, A.; Lee, R.; Hanjani, N. M.; Dehaas, D. L.; Smalley, R. E. Phys. Rev. B 1997, 55, 4921.

(16) Hone, J.; Llaguno, M. C.; Nemes, N. M.; Johnson, A. T.; Fischer, J. E.; Walters, D. A.; Casavant, M. J.; Schmidt, J.; Smalley, R. E. Appl. Phys. Lett. 2000, 77, 666.

(17) Zhang, X. F.; Sreekumar, T. V.; Liu, T.; Kumar, S. J. Phys. Chem. B 2004, 108, 16435.

(18) Zhang, M.; Fang, S. L.; Zakhidov, A. A.; Lee, S. B.; Aliev, A. E.; Williams, C. D.; Atkinson, K. R.; Baughman, R. H. Science 2005, 309, 1215.

(19) Ajayan, P. M.; Schadler, L. S.; Giannaris, C.; Rubio, A. Adv. Mater. 2000, 12,750 .

(20) Yu, M. F.; Files, B. S.; Arepalli, S.; Ruoff, R. S. Phys. Rev. Lett. 2000, 84, 5552.

(21) Kis, A.; Csanyi, G.; Salvetat, J. P.; Lee, T. N.; Couteau, E.; Kulik, A. J.; Benoit, W.; Brugger, J.; Forro, L. Nat. Mater. 2004, 3, 153.

(22) Qian, D.; Liu, W. K.; Ruoff, R. S. Compos. Sci. Technol. 2003, 63, 1561.

(23) Zhu, H. W.; Xu, C. L.; Wu, D. H.; Wei, B. Q.; Vajtai, R.; Ajayan, P. M. Science 2002, 296, 884.

(24) Li, Y. L.; Kinloch, I. A.; Windle, A. H. Science 2004, 304, 276.

(25) Zhang, M.; Atkinson, K. R.; Baughman, R. H. Science 2004, 306, 1358

\section{NL070915C}

